UDC 621.039.624

\title{
3D COILS FOR A COMPACT MIN $B$ MIRROR FIELD WITH MINIMAL FLUX TUBE ELLIPTICITY
}

\author{
O. Agren ${ }^{1}$, V.E. Moiseenko ${ }^{2}$
}

${ }^{1}$ Uppsala University, Uppsala, Sweden

${ }^{2}$ Institute of Plasma Physics, National Science Centre Kharkiv Institute of Physics and Technology, Kharkiv, Ukraine

We determine coil arrangements for reproducing a minimum- $B$ mirror magnetic field, optimized with respect to plasma stability, plasma cross-section ellipticity and particle drift surfaces. The reproduction has to be done with precision, as field errors may give rise to plasma instabilities or collisionless plasma losses due to the guiding centres' drift away from the confinement region. We have developed a set of twisted «fishbone» coils to allow an array of coils to be flexibly stacked, as required for a precise magnetic field reproduction. Results suggest that high mirror ratios of around 10 can be obtained using a fishbone coil arrangement. The mirror ratio can be further increased by finite plasma beta. Parameters representative of a compact $10 \mathrm{MW}$ fusion neutron source have been derived.

Key words: biasing end plates, magnetic mirror, mirror machine, hybrid reactor, fusion neutron source, minimum $B$.

DOI: $10.21517 / 0202-3822-2021-44-2-118-123$

\section{ТРЁХМЕРНЫЕ КАТУШКИ ДЛЯ ПОЛУЧЕНИЯ КОМПАКТНОГО МАГНИТНОГО ПОЛЯ С МИНИМАЛЬНОЙ ИНДУКЦИЕЙ И МИНИМАЛЬНОЙ ЭЛЛИПТИЧНОСТЬЮ ТРУБОК МАГНИТНОГО ПОТОКА ПЛАЗМЫ В ТЕРМОЯДЕРНЫХ УСТАНОВКАХ С МАГНИТНЫМИ ПРОБКАМИ}

\author{
O. Агрен ${ }^{1}$, В.Е. Моисеенко
}

\author{
${ }^{1}$ Университет Уппсалы, Уппсала, Швеция \\ ${ }^{2}$ Институт физики плазмы, Национальный научный центр «Харьковский физико-технический институт», Харьков, Украина
}

Мы определяем расположение трёхмерных катушек в пробкотроне с минимум $B$, оптимизированного с точки зрения стабильности плазмы, эллиптичности поперечного сечения плазмы и поверхностей дрейфа частиц. Воспроизведение должно быть выполнено с большой точностью, так как ошибки поля могут привести к нестабильности плазмы или бесстолкновительным потерям плазмы из-за дрейфа направляющих центров из области удержания плазмы. Мы разработали твистированные катушки «фишбон», которые позволяют гибко укладывать массив катушек в соответствии с требованиями точного воспроизведения магнитного поля. Результаты расчётов показывают, что высокие пробочные отношения около 10 могут быть получены с помощью катушек «фишбон». Пробочное отношение может быть дополнительно увеличено за счёт конечного значения бета плазмы. Получены параметры, характерные для компактного источника термоядерных нейтронов мощностью 10 МВт.

Ключевые слова: смещающие торцевые пластины, пробкотрон, установка с магнитными пробками, гибридный реактор, источник термоядерных нейтронов, минимальная индукция магнитного поля.

\section{INTRODUCTION}

A minimum- $B$ mirror field, optimized with respect to plasma stability, plasma cross-section ellipticity and

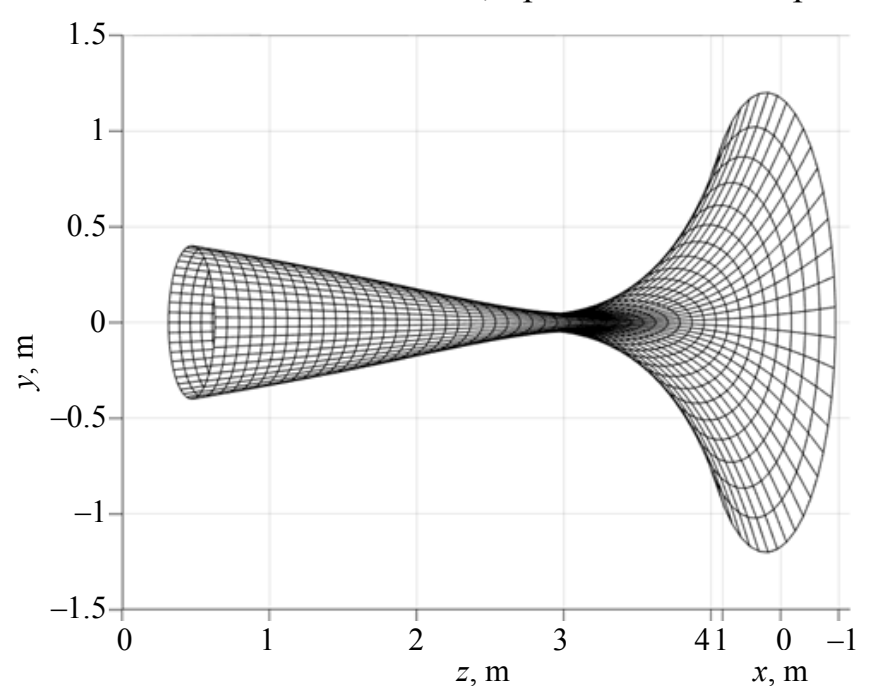

Fig. 1. Magnetic surface of a compact optimized minimum- $B$ field particle drift surfaces has been derived in [1], Fig. 1 (the confinement region has essentially straight and nonparallel magnetic field lines). The shape of the magnetic surface cross section evolves from circular at the mid plane to elliptical near the magnetic field maximum and regains a circular shape at the expander tank wall, where ring-shaped end plates biased to different voltages could be placed. The plot is for a case, where $a=40 \mathrm{~cm}$ (plasma radius), $c_{L}=4 \mathrm{~m}$ (longitudinal scale length), $R_{m}=4$ (mirror ratio) and $M=3$ (magnification of the flux tube cross sectional area at the end tank). In the present paper, we investigate how such a target field can be reproduced by some appropriate arrangement of superconducting 3D-coils. The reproduction of the magnetic field 
needs to be quite precise, since field errors may trigger plasma instabilities or cause collisionless plasma losses by guiding centers drifting away from the confinement region. Straight Ioffe bars and variants of baseball coils and ying-yang coils have been used to create a quadrupolar mirror magnetic field, but the flexibility to shape the magnetic field in more detail is limited, if the coil design does not permit a flexible stacking of many coils. This study is an extension of the research reported in [2], where a special set of «fishbone coils» was introduced for a quadrupolar minimum- $B$ field. We will use a slight modification of those coils in the present study, and expand the parameter range for the reproduction of magnetic field and present a convenient way to make a good precision fit to the target field. Some tasks of interest are constraints set by the coils on device size and the mirror ratio. The mirror ratio should preferably be as high as possible within tolerable limits for the flux tube cross section ellipticity.

\section{COIL MODEL}

The magnetic field from filamentary currents is determined by the Biot-Savart's law:

$$
\mathbf{B}(\mathbf{x})=\frac{\mu_{0} I}{4 \pi} \oint \frac{d \mathbf{l}^{\prime} \times \mathbf{R}}{R^{3}},
$$

where $\mathbf{R}=\mathbf{x}-\mathbf{x}^{\prime}$ is the relative vector between the field point and filament source point. Consider first a single filamentary current with line element $d \mathbf{l}^{\prime}=d \varphi^{\prime}\left(\hat{\boldsymbol{\varphi}}^{\prime} r^{\prime}+\hat{\mathbf{z}} \frac{d z^{\prime}}{d \varphi^{\prime}}\right)$ wound on a round cylinder at distance $r^{\prime}$ from the $z$ axis with a line element: $\quad d \mathbf{l}^{\prime}=\hat{\boldsymbol{\varphi}} r^{\prime} d \varphi^{\prime}+\hat{\mathbf{z}} d z^{\prime}=\left(-y^{\prime} d \varphi^{\prime}, x^{\prime} d \varphi^{\prime}, d z^{\prime}\right), z^{\prime}\left(\varphi^{\prime}\right)=z_{0}^{\prime}+h \cos 2 \varphi^{\prime}$, $d z^{\prime}=\frac{d z^{\prime}}{d \varphi^{\prime}} d \varphi^{\prime}=-2 h \sin 2 \varphi^{\prime} d \varphi^{\prime}$. Here $x^{\prime}=r^{\prime} \cos \varphi^{\prime}, y^{\prime}=r^{\prime} \sin \varphi^{\prime}$ and $\left(r^{\prime}, \varphi^{\prime}, z^{\prime}\right)$ are the cylindrical coordinates for source points on the magnetic axis of the linear device. A finite $h$ gives a quadrupole field component. Compared to the coil set in [2], the straight parts connected by curved elements in [2] are here replaced by the wiggle $z^{\prime}=z_{0}^{\prime}+h \cos 2 \varphi^{\prime}$, which can be anticipated to give a similar contribution to the quadrupolar field. Near the magnetic axis, the Cartesian magnetic field components from this current loop are determined by $B_{z}=f(z)+\mathrm{O}\left(r^{2}\right), \quad B_{x}=\frac{x}{2}\left[-\frac{d f}{d z}+g(z)\right]+\mathrm{O}\left(r^{3}\right), B_{y}=\frac{y}{2}\left[-\frac{d f}{d z}-g(z)\right]+\mathrm{O}\left(r^{3}\right)(1 \mathrm{a}, \mathrm{b}, \mathrm{c})$.

For a single filament we obtain $f(z)=\frac{\mu_{0} I}{4 \pi} r^{\prime 2} \oint \frac{d \varphi^{\prime}}{R_{0}^{3}} g(z)=\frac{\mu_{0} I}{4 \pi} r^{\prime 2} \oint\left(-3 h \frac{1-\cos 4 \varphi^{\prime}}{R_{0}^{5}}-\frac{\partial}{\partial z} \frac{\cos 2 \varphi^{\prime}}{R_{0}^{3}}\right) d \varphi^{\prime}$.

A simple check confirms that these formulas yield $\nabla \cdot \mathbf{B}=0$. The special case of $g(z)=0$ corresponds to an axisymmetric field.

We extend these calculations to a set of filamentary currents $I_{j k}$ wound on cylinders, where $j$ enumerates the cylinder radii and $k$ counts the filament coils on each cylinder surface, compare Fig. 2. Separation distance $r_{j}^{\prime}$ between the cylinder surfaces and the $z$ axis can be written as $r_{j}^{\prime}=a_{0}+j \Delta r^{\prime}, j=0,1,2, \ldots, N_{r}$, where $a_{0}$ is

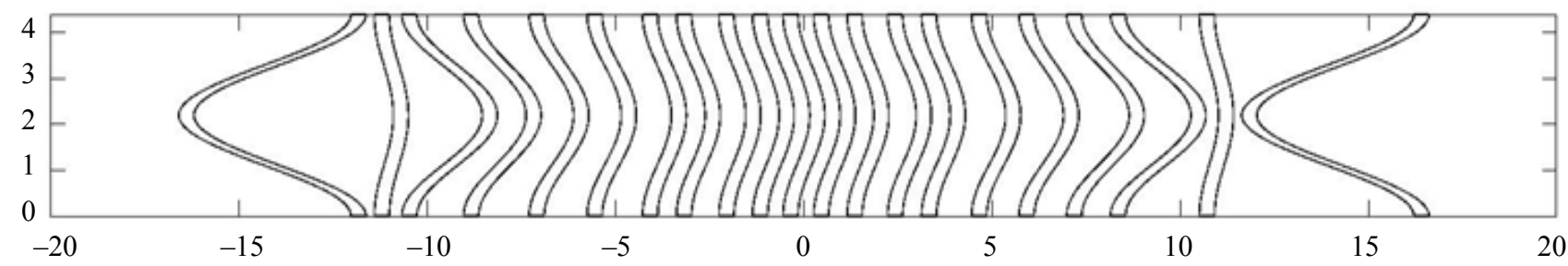

Fig. 2. Coil boundaries versus arc length $a_{0} \varphi^{\prime}$ at the inner coil radius, which is the radius where the filament curvatures are at their maximum. Measurement units are meters. The complete coil winding includes analogous layers of wire currents at constant external radii $r_{j}^{\prime}$ wound with the same variation with $\varphi^{\prime}$, which facilitates convenient stacking of the coils. Each coil layer at a constant radius has a width $L_{z}$ along $z$, and a package is formed by adding such layers in the interval of $a_{0} \leq r_{j}^{\prime} \leq a_{1}$, where $a_{1}$ is the outer coil radius. The coil set consists of a sequence of pair of identical coils on the opposite sides of the mid plane at $z=0$. The figure is for the case, where a coil pair closest to the mid plane has coalesced in to a single coil 
the inner radius and $a_{1}=a_{0}+N_{r} \Delta r^{\prime}$ is the outer radius of the coil package. Filamentary currents with currents $I_{j k}$ are wound at the cylindrical radius $r_{j}^{\prime}$ with the axial source coordinates (compare Fig. 2): $z_{k}^{\prime}\left(\varphi^{\prime}\right)=z_{0, k}^{\prime}+h_{k} \cos 2 \varphi^{\prime}$, $k= \pm 1, \pm 2, \ldots, k_{\max }$. Here $z_{0, k}^{\prime}$ determines the axial center of the filamentary current. For simplicity, we take $I_{j k}=I_{k}$ for all $j$ values (for convenient stacking of the coils, we will arrange packages, where all filamentary currents in a given package have the same $z_{0, k}^{\prime}, h_{k}$ and $I_{k}$ values). The described winding results in $1, g(z)=\sum_{j, k} B_{k} \cdot \frac{g_{j, k}(z)}{a_{0}}$, where $B_{k}=\mu_{0} I_{k} /\left(2 a_{0}\right)$ and $f_{j k}(z)=\frac{a_{0} r_{j}^{\prime 2}}{2 \pi} \oint \frac{d \varphi^{\prime}}{R_{0, j k}^{3}}, g_{j, k}(z)=\frac{a_{0}^{2} r_{j}^{\prime 2}}{2 \pi} \oint\left(-3 h_{k} \frac{1-\cos 4 \varphi^{\prime}}{R_{0, j k}^{5}}-\frac{\partial}{\partial z} \frac{\cos 2 \varphi^{\prime}}{R_{0, j k}^{3}}\right) d \varphi^{\prime}$. With $z_{0,-k}^{\prime}=-z_{0, k}^{\prime}$, $h_{-k}=h_{k}$ and $I_{j,-k}=I_{j, k}$, we have a symmetric quadrupole field satisfying $f(-z)=f(z)$ and $g(-z)=g(z)$.

Details of the coil arrangement will be presented elsewhere. As indicated in Fig. 2, for convenient stacking, we collect filaments into paired packages, where parameters for the current $I_{i}$ the quadrupolar parameters $h_{i}$ are identical. Each package, which models a multi-turn winding in a cryogenic coil, has width $L_{z}$ along the $z$ direction, compare Fig. 2, and identical inner and outer coil radii. With an arrangement where identical coils are placed on opposite sides of the mid plane, a symmetric minimum- $B$ field is formed, satisfying $f(-z)=f(z)$ and $g(-z)=g(z)$. Overlapping between the coils must be avoided.

\section{TARGET MAGNETIC FIELD PROPERTIES}

With $B_{0}=f(0)$, we introduce $u_{e}(z)$ and $\gamma_{0}(z)$ by $u_{e}(z)=\sqrt{\frac{B_{0}}{f(z)}} \cosh \left(\int_{0}^{z} \frac{g}{2 f} d z\right), \gamma_{0}(z)=\sqrt{\frac{B_{0}}{f(z)}} \sinh \left(\int_{0}^{z} \frac{g}{2 f} d z\right)$, where $u_{e}(z)=u_{e}(-z)$, and is an even function and $\gamma_{0}(z)=-\gamma_{0}(-z)$ is an odd function. In the equatorial plane, these functions satisfy the Cauchy conditions, namely, $u_{e}(0)=1, \gamma_{0}(0)=0, u_{e}^{\prime}(0)=0$ and $\gamma_{0}^{\prime}(0)=1 / c_{L}$, where $c_{L}=2 f(0) / g(0)$ is a parameter, which determines the longitudinal length scale for the quadrupolar field (axisymmetric fields correspond to $c_{L} \rightarrow \infty$ ). The Cartesian components of the field are in the paraxial approximation determined by $B_{z}=\frac{B_{0}}{u_{e}^{2}-\gamma_{\mathrm{o}}^{2}}, B_{x}=x \frac{B_{0}}{u_{e}^{2}-\gamma_{\mathrm{o}}^{2}} \frac{u_{e}^{\prime}+\gamma_{\mathrm{o}}^{\prime}}{u_{e}+\gamma_{\mathrm{o}}}, B_{y}=y \frac{B_{0}}{u_{e}^{2}-\gamma_{\mathrm{o}}^{2}} \frac{u_{e}^{\prime}-\gamma_{\mathrm{o}}^{\prime}}{u_{e}-\gamma_{\mathrm{o}}}$.

Near $z=0$ this reduces to $B_{x} \rightarrow\left(x / c_{L}\right) B_{0}$ and $B_{y} \rightarrow\left(y / c_{L}\right) B_{0}$. The formulas show that the shape of the magnetic field near the $z$ axis is determined by $u_{e}(z)$ and $\gamma_{0}(z)$. Our goal is to fit $u_{e}(z)$ and $\gamma_{0}(z)$ to the target functions $u_{e}^{(\mathrm{SF})}(z)$ and $\gamma_{\mathrm{o}}^{(\mathrm{SF})}(z)$, derived in [1] for an optimized minimum- $B$ field. The target functions correspond to an extended SFLM (straight field line mirror) field with a minimal flux surface ellipticity for a given mirror ratio, which will be connected to expanding flux tubes beyond the mirror throats. Over most of the confinement region, apart from a close vicinity to the location for the maximal magnetic field strength, the corresponding target functions are accurately described by the simple SFLM expressions $u_{e}^{(\mathrm{SF})}(z)=1$ and $\gamma_{0}^{(\mathrm{SF})}(z)=z / c_{L}$, which sim-

plifies the fitting in that region. This ideal SFLM field results in: $B \rightarrow \frac{B_{0}}{1-z^{2} / c_{L}^{2}}$, allowing desirable properties for a region with a finite mirror ratio to be obtained, although this simple solution cannot be directly extended to the expander region beyond the mirror throats due to singularities that show up at $z= \pm c_{L}$. The extended SFLM field derived in [1] is somewhat (but importantly) deviated from the ideal SFLM solution in the confinement region, which enables a smooth transition into the end tank region. The magnetic field from the coils should also provide suitable properties on the path to the expander tank region. 
We use the SFLM target functions derived in $[1]: \quad \gamma_{0}^{(\mathrm{SF})}(z)=\frac{z}{c_{L}}\left[1-\frac{1}{4\left(1+\varepsilon_{N}\right)}\left(\frac{\kappa_{0} z}{c_{L}}\right)^{2 N}\right]$, $u_{\mathrm{e}}^{(\mathrm{SF})}(z)=1+\frac{1}{4\left(1-\varepsilon_{N}\right)}\left(\frac{\kappa_{0} z}{c_{L}}\right)^{2 N}$. Here $\varepsilon_{N}=1 /(2 N)$, integer $N$ determines how close the target function is to the idealised SFLM field, and mirror ratio $R_{m}$ determines the $\kappa_{0}$ parameter. With $R_{m}=4$ and $N=4, \kappa_{0}=0.839$, and parameter values for other cases can be found in [1]. The above target functions are only applicable to confinement region $-z_{B} \leq z \leq z_{B}$, where the magnetic field strength is at its maximum at the end points. Apart from the small, but important, deviations, we have $u_{e}^{(\mathrm{SF})}(z)=1$, and $\gamma_{\mathrm{o}}^{(\mathrm{SF})}(z)=z / c_{L}$ in the confinement region. The coil parameters should demonstrate a proper magnetic field evolution into the end tank region beyond the mirror throats. Our goal is to provide desired flux tube magnification $M$ at the end tank wall with nearly circular flux surface footprints at the end tank to avoid short circuiting between the end plates, which are biased to different voltages. Nearly circular shapes will certainly appear far enough beyond the mirrors, since the vacuum field asymptotically approaches an axisymmetric dipole field.

To generate the magnetic field, we first select parameters for mirror ratio $R_{m}$ and longitudinal scale length $c_{L}$, together with coil parameters for the inner coil radius $a_{0}$, the outer coil radius $a_{1}$, and their axial length, $L_{z}$. We then do the fitting by selecting $i_{\max }$, i.e. the number of coil package pairs, and adjusting the coil parameters to match current $I_{i}$, wiggling $h_{i}$ and central coil position $z_{0, i}$ for each coil, so that apart from very small field errors, we end up with $u_{e}(z)=u_{e}^{(\mathrm{SF})}(z)$, and $\gamma_{\mathrm{o}}(z)=\gamma_{\mathrm{o}}^{(\mathrm{SF})}(z)$ in the confinement region. An almost exact agreement with the target function assures that the coils produce a magnetic field satisfying the minimum- $B$ criterion near the axis, which corresponding MHD stability for the flute and ballooning modes, accompanied with favourable properties for flux tube ellipticity and suppression of neoclassical transport effects.

\section{RESULT FOR A FUSION NEUTRON SOURCE}

As an example, we have considered a case which could be representative for a fusion neutron source with a fusion power in the range of $10 \mathrm{MW}$. In Figs. 2 and 3, plasma dimension parameters are $a=0.35 \mathrm{~m}$ and $c_{L}=10 \mathrm{~m}$. The coil parameters are $a=1.4 \mathrm{~m}$, and $L_{z}=0.4 \mathrm{~m}$. The available width for shielding and other arrangements can be estimated from $\Delta a_{\text {shyield }} \approx a_{0}-2 a=70 \mathrm{~cm}$. For this case, the reproduction was made using mirror ratio $R_{m}=6$ and parameter value $a_{0} / c_{L}=0.14$. To match the strong gradients of the target functions for this rather high mirror ratio, it was necessary to consider a low value of $a_{0} / c_{L}$. The target functions (with $N=3$ ) and the coil fitting give a good agreement in the confinement region as seen in Fig. 3.

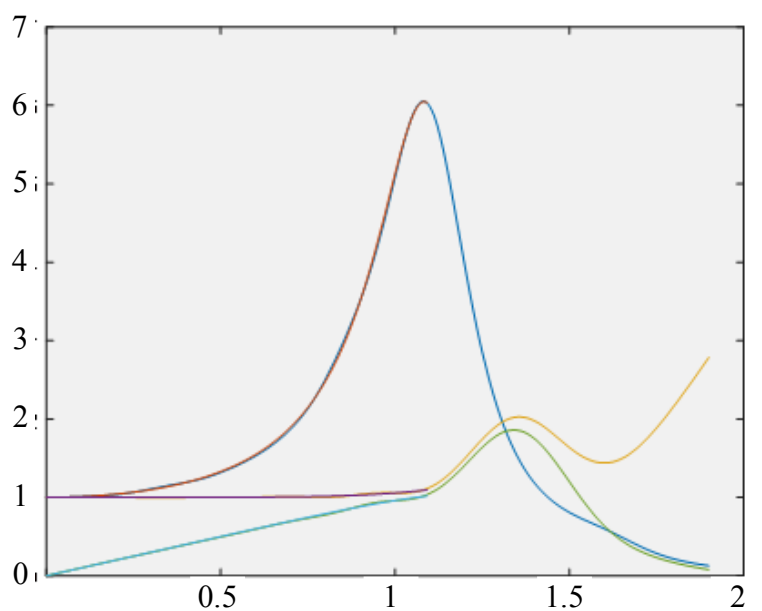

Fig. 3. The $u_{e}(z), \gamma_{0}(z)$, functions (yellow and green, respectively) and the corresponding mirror ratio $R_{m}(z)=1 /\left(u_{\mathrm{e}}^{2}-\gamma_{\mathrm{o}}^{2}\right)$ (blue) generated by the coil set versus $z / c_{L}$ is shown. The plots demonstrate accurate reproductions of the SFLM field in the confinement region

The coil parameters

\begin{tabular}{c|c|c|c|c|c|c|c|c|c|c|c}
\hline$i$ & 1 & 2 & 3 & 4 & 5 & 6 & 7 & 8 & 9 & 10 & 11 \\
\hline $\mathrm{z}_{0, i}^{\prime} / c_{L}$ & 0 & 0.081 & 0.17 & 0.28 & 0.37 & 0.51 & 0.65 & 0.8 & 0.94 & 1.0946 & 1.41 \\
$h_{1} / a_{1}$ & -0.252 & -0.252 & -0.252 & -0.272 & -0.272 & -0.322 & -0.422 & -0.592 & -0.762 & -0.18 & 1.63 \\
$\Delta \mathrm{z}_{i}^{\prime}, \mathrm{cm}$ & - & 41 & 49 & 67.2 & 50 & 93 & 86 & 86.2 & 76.2 & 33.12 & 22 \\
$\tilde{I}_{i}$ & 0.1239 & 0.2767 & 0.3346 & 0.3139 & 0.4543 & 0.5369 & 0.7022 & 0.5782 & 2.3336 & 6.3276 & 0.9491
\end{tabular}

(Note that separations $\Delta z_{i}^{\prime}$ between neighbouring coils provide space for the structural support and openings to the vacuum chamber. The $\tilde{I}_{i}$ parameters represent dimensionless coil currents.) 
The ellipticity at a maximum $B$ strength is reasonably well described by a simple estimate $\varepsilon_{\text {ell }}\left(R_{m}\right) \approx 4 R_{m}$. Because the target vacuum field is close to a marginal minimum- $B$ field, we need to assure that field errors do not give rise to plasma instabilities. The small field errors obtained here are unlikely to pose any problem for flute stability, since they would be surpassed by several other stabilizing effects. Although the gas-dynamic regime may, in principle, provide stabilization for an expander with its favourable curvature, other mechanisms of stability control should be identified, if we aim for a more collision-free regime. Line tying could be one such mechanism. Wall stabilization is present since a plasma displacement induces image currents in a conducting vacuum vessel. Most importantly, a finite beta deepens the magnetic well and is therefore expected to strongly improve flute stability. Small field errors also are not expected to have any dramatic effect on the confinement, if a radial electric field is applied, since the $\mathbf{E} \times \mathbf{B}$ rotation tends to smear out guiding centre excursions caused by the field errors. There are therefore reasons to anticipate a robust confinement in the magnetic field with favourable MHD-stability properties.

We have also computed the representations of a more compact test device with a 6-m long confinement region, as well as a full scale stand-alone fusion reactor with a 100-m long confinement region. The 6-m long device could be of interest for testing purposes, if optimal confinement properties could be demonstrated in a reallife device, and such a device could also be designed for investigations of centrifugal confinement, where a controlled $\mathbf{E} \times \mathbf{B}$ rotation provides a plasma heating source. Apparently, centrifugal confinement schemes [4, 5] have not yet been tested on a device with superior MHD-stability properties, such as the SFLM field, where stability of flute and ballooning modes is predicted. An interesting possibility with the centrifugal confinement is the potential for counteracting pitch angle scattering, since it could provide a torque and a corresponding kinetic energy deposition into perpendicular velocity components. Since only a small tilt in pitch angle is required to prevent a particle from escaping into the loss cone, there may be a chance that the longitudinal confinement in mirrors is dramatically increased with a moderate power applied to the centrifugal confinement scheme. If so, a mirror may even be relevant as a stand-alone fusion reactor.

A 100-m long full scale device can be designed with a mirror ratio exceeding 10 for the vacuum field using our magnetic shaping method. Including a finite plasma $\beta$, there may be a possibility to reach a mirror ratio of around 20 [1]. A good confinement of energetic particles is anticipated in the SFLM field, while the confinement of tritium and Helium 3 particles from the D-D-fusion reaction is expected to substantially increase the power output from a D-D-plasma. Several crucial problems concerning tritium inventory and its breading ratio could be eased by a fusion device using the D-D-fuel, however that would require a by far higher confinement than achieved in any device developed so far. It seems plausible that commercial fusion energy development will be confined to one or another scenario involving only the D-D-fuel cycle.

Essentially, a target field can be reproduced to a certain maximal mirror ratio, where higher values for the mirror ratio are obtainable for long-thin systems. For a sufficiently long-thin system, mirror ratios in the range of 10 can be achieved. Finite plasma $\beta$ can be exploited to raise the mirror ratio to around 20.

\section{SUMMARY AND DISCUSSION}

A detailed reproduction of an optimized magnetic field is necessary to avoid plasma instabilities. MHD stability for both flute and ballooning modes with $\beta \sim 1$, combined with minimal ellipticity, are envisaged for the SFLM field. Arrangements can also be made to suppress the neoclassical transport effects. The objective of this study was to identify a practical and convenient coil set for the reproduction of a SFLM field, and to determine the limiting values of the mirror ratio for this coil set. Our fishbone coil arrangement can be applied to a wide range of sizes for a minimum- $B$ mirror field.

The most critical parameter for accurate reproduction is ratio $\frac{a_{0}}{c_{L}}=\frac{\text { inner coil radius }}{\text { axial scale length }}$.

It determines the upper limit on the mirror ratio, and higher mirror ratios can be fitted with smaller values of $a_{0} / c_{L}$. For a sufficiently long-thin system, we can achieve $R_{m} \approx 10$ for the vacuum field, with tolerable values for the ellipticity. With a finite plasma beta, the mirror ratio can be increased further. In a minimum- $B$ vacuum magnet field, a finite $\beta$ would not trigger flute instability (because diamagnetic currents deepen the magnetic 
well) or increase the ellipticity. For the optimised magnetic field, the largest flux tube ellipticity in the confinement region is estimated as $\varepsilon_{\text {ell }} \approx 4 R_{m}(0)$, where $R_{m}(0)$ is the vacuum field mirror ratio.

The fishbone coils are smoothly twisted, which is favourable for superconductors [3]. Results representative for a $10 \mathrm{MW}$ fusion neutron source are presented in this paper. A detailed reproduction of the target SFLM field is also possible for a wider parameter range.

A more compact magnetic field design may be appropriate for a 6-m long test device, where the existence of the radial invariant could be tested experimentally. The idea is that a quasi-neutral electric field, controlled by biased end plates, would act like a «glue» to force each guiding centre to move in close vicinity of its mean magnetic surface, which is connected with the radial constant of motion [1]. The latter prevents a collision-free radial leakage, allowing the neoclassical transport effects to be neglected.

With higher (around $100 \mathrm{kV}$ ) bias voltages, there may also be the possibility of testing the centrifugal confinement $[4,5]$, as well as plasma heating by a controlled $\mathbf{E} \times \mathbf{B}$ rotation. The point is to test this heating scenario for a magnetic field with superior MHD-stability properties. To the best of our knowledge, experiments performed so far on other devices have been using magnetic field designs prone to MHD-instabilities.

\section{REFERENCES}

1. Ågren O., Moiseenko V.E. — Fusion Engineering and Design, 2020, vol. 161, p. 111943.

2. Hagnestål A., Ågren O., Moiseenko V.E. - J. Fusion Energ., 2012, vol. 31, p. 379.

3. Chaddah, P. Critical current densities in superconducting materials. - Sadhana, 2003, vol. 28, p. 273-282; https://doi.org /10.1007/BF02717137.

4. Ellis R.F., Hassam A.B., Messer S., Osborn B.R. — Physics of Plasmas, 2001, vol. 8, p. 2057; https://doi.org/10.1063/1.1350957.

5. Volosov V.I., Pekker M.S. — Nucl. Fusion, 1981, vol. 21, p. 1275.

\section{AUTHORS}

O. Ågren; Uppsala University, Ångström Laboratory, Box 534, SE-751 21 Uppsala, Sweden, olov.agren@angstrom.uu.se V.E. Moiseenko, Institute of Plasma Physics, National Science Center KIPT, 61108 Kharkiv, Ukraine, moiseenk@ipp.kharkov.ua 